\title{
ALTERATIONS IN POLYRIBOSOMES ASSOCIATED WITH DENDRITIC SPINES DURING THE REINNERVATION OF THE DENTATE GYRUS OF THE ADULT RAT ${ }^{1}$
}

\author{
OSWALD STEWARD ${ }^{2}$
}

Departments of Neurosurgery and Physiology, University of Virginia School of Medicine, Charlottesville, Virginia 22908

Received March 30, 1982; Revised July 15, 1982; Accepted July 19, 1982

\begin{abstract}
Many dendritic spines in the dentate gyrus normally have polyribosomes under the spine base. The present study asks whether there are changes in these spine-associated polyribosomes when the spines are denervated and reinnervated following lesions. Rats were prepared for electron microscopy $2,4,6,8,10,12$, and 14 days, and approximately 7 months following unilateral destruction of the entorhinal cortex. Dendritic polyribosomes were evaluated in the denervated neuropil of the dentate gyrus ipsilateral to the lesion and in the neuropil of the contralateral (control) dentate gyrus. Qualitative observations revealed that polyribosomes in denervated dendrites were selectively localized under the base of dendritic spines as is also the case in normal dendrites. However, the incidence of polyribosomes under the base of spines appeared qualitatively much higher than normal, and there appeared to be a substantial increase in the number of spine necks and heads with polyribosomes. Quantitative analyses were undertaken to evaluate (1) the incidence of polyribosomes under spine bases, (2) the number of polyribosome-containing spine necks and heads per area of neuropil, and (3) the total number of polyribosomes within dendritic segments within the denervated zone. The incidence of polyribosomes under spine bases was evaluated in each animal from a series of 21 to 22 photographs of identified spines (a spine visible in its entirety from its point of origin on the parent dendrite to the region of synaptic contact). All identified spines within each photographic field of approximately $60 \mu \mathrm{m}^{2}$ were scored for the presence of polyribosomes. Over the post-lesion interval, the incidence of polyribosomes associated with identified spines increased about 3-fold from the control incidence of $11.6 \%$ (determined from a similar series of photographs from the control side of each animal). Peak incidence was observed at 8 days post-lesion. The number of polyribosomecontaining spine bases per $1000 \mu \mathrm{m}^{2}$ of neuropil increased with the same time course, indicating that the changes in incidence were not a result of a selective disappearance of spines without polyribosomes. The number of polyribosome-containing spine necks and heads per $1000 \mu \mathrm{m}^{2}$ of neuropil was defined in the same series of photographs and also increased about 3 -fold, with approximately the same time course as the increases in polyribosome-containing spine bases. Despite the dramatic increases in the number of polyribosomes in and around spines, there was only a very modest increase in the total number of dendritic polyribosomes per area of neuropil and essentially no increase in the number of polyribosomes per area of dendrite. The combined results suggest a redistribution of dendritic polyribosomes into the region of the dendrite which is specialized for the receipt of an afferent synapse (the spine and its postsynaptic membrane specialization). This redistribution occurs during a period of terminal proliferation, suggesting that these two processes are related. I propose that these events reflect some process within the denervated dendrites related to the preparation of the dendrite for reinnervation or to the initiation of the sprouting response.
\end{abstract}

We have recently reported that many spines on granule cells of the rat's dentate gyrus have accumulations of

\footnotetext{
'I thank S. L. Vinsant for her excellent technical help, R. A. Ogle for help in preparing the illustrations, and B. Fass for his helpful comments on early versions of the manuscript. This work was supported by National Institutes of Health Grant NS12333. O. S. is the recipient
}

polyribosomes under their base (Steward and Levy, 1982). Indeed, most of the polyribosomes within granule

of Research Career Development Award NS00325.

${ }^{2}$ To whom correspondence should be addressed at Department of Neurosurgery, University of Virginia School of Medicine, Box 420, Charlottesville, VA 22908. 
cell dendrites are selectively localized under spine bases, leading us to speculate that they might be involved in the synthesis of some protein(s) specifically related to the spine or the synapse. It was therefore of considerable interest to determine whether there were changes in these spine-associated polyribosomes during a period of synapse construction. One way to approach this question is to take advantage of lesion-induced synapse turnover. In the rat, the destruction of the ipsilateral entorhinal cortex removes approximately 85 to $90 \%$ of the normal synapses on the distal portions of granule cell dendrites (Matthews et al., 1976). Over time, these synapses are replaced as a result of the proliferation of surviving presynaptic processes and the formation of new synapses with the denervated dendrites (see Matthews et al., 1976). The proliferation of the presynaptic elements occurs between about 6 and 10 days post-lesion, based on light microscopic studies of specific afferent systems (Lynch et al., 1977; Steward and Loesche, 1977) and on the basis of electron microscopic counts of presynaptic profiles (Steward and Vinsant, 1982). The formation of synapses with denervated dendrites seems to occur later, with most of the reinnervation occurring after 10 days postlesion (see Matthews et al., 1976, and for a detailed description of the time course of presynaptic growth and reinnervation, see Steward and Vinsant, 1982). This phenomenon of lesion-induced synapse turnover thus presents an ideal opportunity to evaluate whether the spineassociated polyribosomes undergo any changes during synaptogenesis.

There are some reasons to believe that an evaluation of potential changes in polyribosomes in response to denervation may contribute to an understanding of the reinnervation process. It has long been known that denervation may result in profound changes in the postsynaptic neurons (see, for example, Jones and Thomas, 1962; Pinching and Powell, 1971; and for a general review, see Globus, 1975). Many of the changes may be degenerative in nature, but some may be related to restorative processes. For example, the postsynaptic cell may need to prepare its membrane surfaces for reinnervation, build new postsynaptic specializations or renovate old sites, rebuild the cytoskeletal scaffolding associated with spines and dendrites, or even elaborate and release substances which affect glia or which induce presynaptic growth (trophic substances). Indeed, some evidence already exists suggesting dynamic changes in spines with denervation and reinnervation (Parnavelas et al., 1974) and even in the main dendrite itself (Caceres and Steward, 1981, 1982). Thus, events within the postsynaptic cell may be permissive or inductive to the reinnervation process.

Because of the possibility that events within the denervated postsynaptic cell might be crucial to the reinnervation process and because of other evidence which suggests an increase in protein precursor incorporation in the denervated dendritic regions during reinnervation (Steward, 1982; Fass and Steward, 1981, 1982), we were particularly interested in evaluating (1) the incidence of polyribosomes under the base of dendritic spines, (2) the number of spines with polyribosomes in the spine neck or head, and (3) the total number of polyribosomes contained within dendritic segments in the denervated zone during the period of terminal proliferation and lesion-induced synaptogenesis. Here, I report that during the period of maximal terminal proliferation (between 6 and 10 days post-lesion) there are dramatic increases in the incidence of polyribosomes in their normal preferred location (under the base of dendritic spines). There are also changes in the distribution of polyribosomes; clusters of polyribosomes are found much more frequently in spine necks and even spine heads, often lying just subjacent to the postsynaptic membrane specialization. There are also modest increases in the total number of polyribosomes within dendrites, although the increases in the total number of dendritic polyribosomes are not as dramatic as the increases in their frequency in and around spines. Preliminary descriptions of some of the results in the present study have appeared in abstract form (Steward and Fass, 1981) and in brief reviews (Steward, 1982; Steward and Fass, 1982).

\section{Materials and Methods}

The experimental animals were male Sprague-Dawleyderived rats. The entorhinal cortex was destroyed unilaterally in each animal, according to procedures described previously (Loesche and Steward, 1977), when the animals were approximately 90 to 150 days of age. The animals were prepared for electron microscopy $2(N=$ 4), 4, 6, 8, $10(N=3), 12$, and 14 days, and approximately 7 months (211 to 218 days) post-lesion (two animals at each survival interval except for 2 and 10 days). This is the same series of animals that was used for a quantitative electron microscopic evaluation of terminal proliferation and synapse replacement in the denervated dentate gyrus (Steward and Vinsant, 1982), except that only 2 of the 4 animals sacrificed 2 days post-lesion were included in the present study. In addition, one of the cases sacrificed at 10 days post-lesion was inadequately fixed for an evaluation of polyribosome distribution and is therefore not included in the present analysis. Thus, a total of 16 animals were included in the present study.

Electron microscopy. The animals were perfused transcardially with a solution of $2 \%$ paraformaldehyde/ $2 \%$ glutaraldehyde in $0.13 \mathrm{M}$ cacodylate buffer $(\mathrm{pH} 7.2)$ while deeply anesthetized with sodium pentobarbital. The brains were removed after being allowed to postfix in situ for 1 to $3 \mathrm{hr}$ at $5^{\circ} \mathrm{C}$ and were postfixed in the perfusion solution for an additional hour. The brains then were blocked by hand into rostral and caudal halves. The caudal half of the brain (including the entorhinal cortex and posterior hippocampal formation) was embedded in egg yolk according to the method of Ebbesson (1970), sectioned at $40 \mu \mathrm{m}$ on a freezing microtome, and stained with cresyl violet to ascertain the extent of the lesion. The rostral half of the brain was sectioned coronally at 150 to $300 \mu \mathrm{m}$ either with a hand-held razor blade or with the aid of an Oxford Vibratome.

The thick coronal sections were collected in cacodylate buffer, and portions of the dentate gyrus ipsilateral and contralateral to the lesion were dissected free hand. From each thick section, the dentate gyrus was divided into dorsal and ventral blades, each of which extended from the layer of dentate granule cells to the tips of the granule 
cell dendrites (the hippocampal fissure in the case of the dorsal blade and the pial surface in the case of the ventral blade).

The sample blocks were osmicated for $45 \mathrm{~min}$ and embedded in Epon/Araldite with routine procedures. Thin sections were cut perpendicular to the layer of granule cell bodies with an LKB ultramicrotome. The sections were mounted on slot grids $(1 \times 2 \mathrm{~mm})$ and stained with methanolic uranyl acetate/lead citrate. Adjacent semi-thin $(1 \mu \mathrm{m})$ sections were mounted on glass slides and stained with toluidine blue.

Because the projection from the entorhinal cortex to the dentate gyrus is almost exclusively ipsilateral in the normal albino rat (Steward et al., 1976; Steward, 1976) the dentate gyrus contralateral to the lesion served as the control. It is important to note, however, that there is a very sparse crossed projection from one entorhinal cortex to the contralateral dentate gyrus (Goldowitz et al., 1975; Steward, 1976). While other studies suggest that only about $5 \%$ of the synapses in the entorhinal terminal zone are from the contralateral side, the "control" material presumably is not entirely normal. Despite this reservation, the sparseness of the crossed projection and the consistency of our quantitative observations on the control side give us confidence that the material which we have analyzed as the control differs little if at all from normal material. The control sides from 4 of the animals (those sacrificed 6 and 8 days post-lesion) were not included in the analysis, however, since these animals also received injections of $\left[{ }^{3} \mathrm{H}\right]$ proline into the surviving entorhinal cortex prior to sacrifice (data not reported). These injections produced slight damage in the entorhinal cortex and resulted in some terminal degeneration in the dentate gyrus ipsilateral to the injection. Thus, the control material was derived from only 11 of the animals.

The electron microscopic observations were carried out in the middle portions of the molecular layer of the ventral blade of the dentate gyrus, from an area approximately 75 to $150 \mu \mathrm{m}$ from the layer of granule cell somata. This portion of the neuropil contains the midproximodistal segments of the granule cell dendrites. The location for sampling was determined solely by distance from the granule cell layer on the control side. On the side ipsilateral to the lesion, an additional criterion was the presence of some terminal degeneration in the field of view, to ensure that the observations were in fact made in the denervated zone. Since destruction of the entorhinal cortex denervates approximately the outer two-thirds of the molecular layer of the ventral blade, the observations on the denervated side were centered in the more proximal portions of the denervated zone. The procedures for sampling and for the quantitative evaluations are described under "Results." Measurements of crosssectional area were carried out on photographic prints (final magnification $\times 27,000$ ) with the aid of a Zeiss Videoplan system.

Throughout the "Results," the term "identified spine bases" will refer to intersections between a main dendritic shaft and a spine neck. The spine neck in turn is identified as such on the basis of (1) being continuous with a spine head which receives a presynaptic contact upon an area of membrane specialization or (2) containing a well de- fined spine apparatus. Polyribosomes are defined as a collection of particles of the size and electron density of ribosomes.

\section{Results \\ Polyribosomes in dendrites in the normal dentate gyrus}

We have previously described the preferential localization of polyribosomes under the base of dendritic spines in the normal dentate gyrus (Steward and Levy, 1982). While there are not large numbers of polyribosomes in dendrites except near the cell soma, the polyribosomes which are present are preferentially localized under the intersection between the spine neck and the main dendritic shaft (see Fig. 1, $A$ and $B$ ) or under mounds in the dendrite which serial sections reveal to be the base of spines. While the majority (about $80 \%$ ) of the polyribosomes in mid-proximodistal dendrites are found under identified or presumed spine bases (mounds), not all spines have polyribosomes. In our previous study, about $12 \%$ of the profiles which could be identified as spines in single sections contained polyribosomes, and in a limited series of 34 through-sectioned spines, 10 or $29 \%$ contained polyribosomes in one or more of the sections comprising the series. Thus, in the normal dentate gyrus, polyribosomes appear to have a special association with some spines, although perhaps not the majority.

\section{Qualitative observations on polyribosomes in denervated/reinnervated dendrites}

With denervation and reinnervation, there are substantial alterations in the morphology of granule cell dendrites which we have described elsewhere. During the early post-lesion interval, the picture is one of dendritic deterioration. Golgi studies reveal a collapse and atrophy of dendrites; the dendrites which survive become tortuous and varicose, and there is a loss of dendritic spines (Caceres and Steward, 1981, 1982). Electron microscopic observations confirm the Golgi studies and also reveal that the spines appear to collapse into the dendrite (Steward and Vinsant, 1982). There also appear to be fewer dendritic microtubules, and the dendrite, particularly at varicosities, contains a flocculent material similar to that normally found in spines. An impression of the changes which occur in denervated dendrites may be gained by comparing the morphology of dendrites from control material (Fig. 1, A and B) with that at 6 to 8 days post-lesion (Figs. 1, $C$ and $D$, and 2). With reinnervation, many of the dendritic changes are reversed in a way that is suggestive of dendritic growth. Specifically, there is an increase in dendritic mass in the denervated zone; the dendrites appear less tortuous and have fewer varicosities, and spine density recovers to near normal levels (Caceres and Steward, 1981, 1982). During the period of reinnervation, even the spines contacted by nondegenerating presynaptic terminals often are quite abnormal. They typically are stubby, and some have relatively thin postsynaptic specializations (See Fig. 2, $C$ and $D$ ). These atypical spines are quite reminiscent of immature spines and spine synapses in developing animals (Bloom, 1972). 

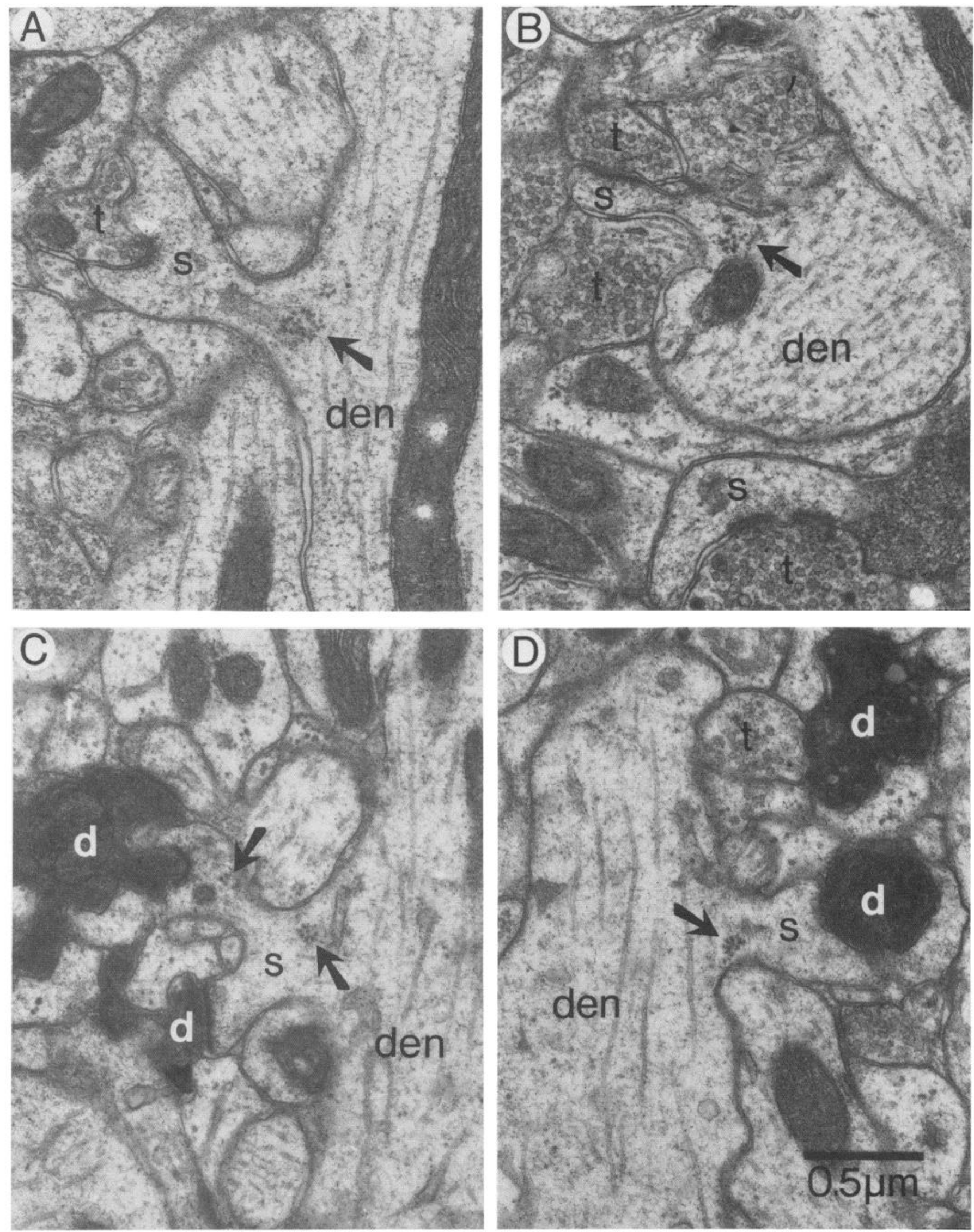

Figure 1. Polyribosomes under dendritic spines. $A$ and $B$ illustrate typical configurations of polyribosomes under the base of dendritic spines in the molecular layer of the normal dentate gyrus. Note the hint of a membranous cistern between the polyribosomes and the spine neck in $A$. $C$ and $D$ illustrate configurations of polyribosomes in the denervated dentate gyrus (6 days post-lesion). Note that the spine in $D$ appears collapsed (other examples of collapsed spines may be found in Steward and Vinsant, 1982, and in Fig. $2 A$ ). $t$, presynaptic terminals; $s$, spines; den, dendrites; $d$, degenerating terminals. The unlabeled arrows point to the polyribosomes. 

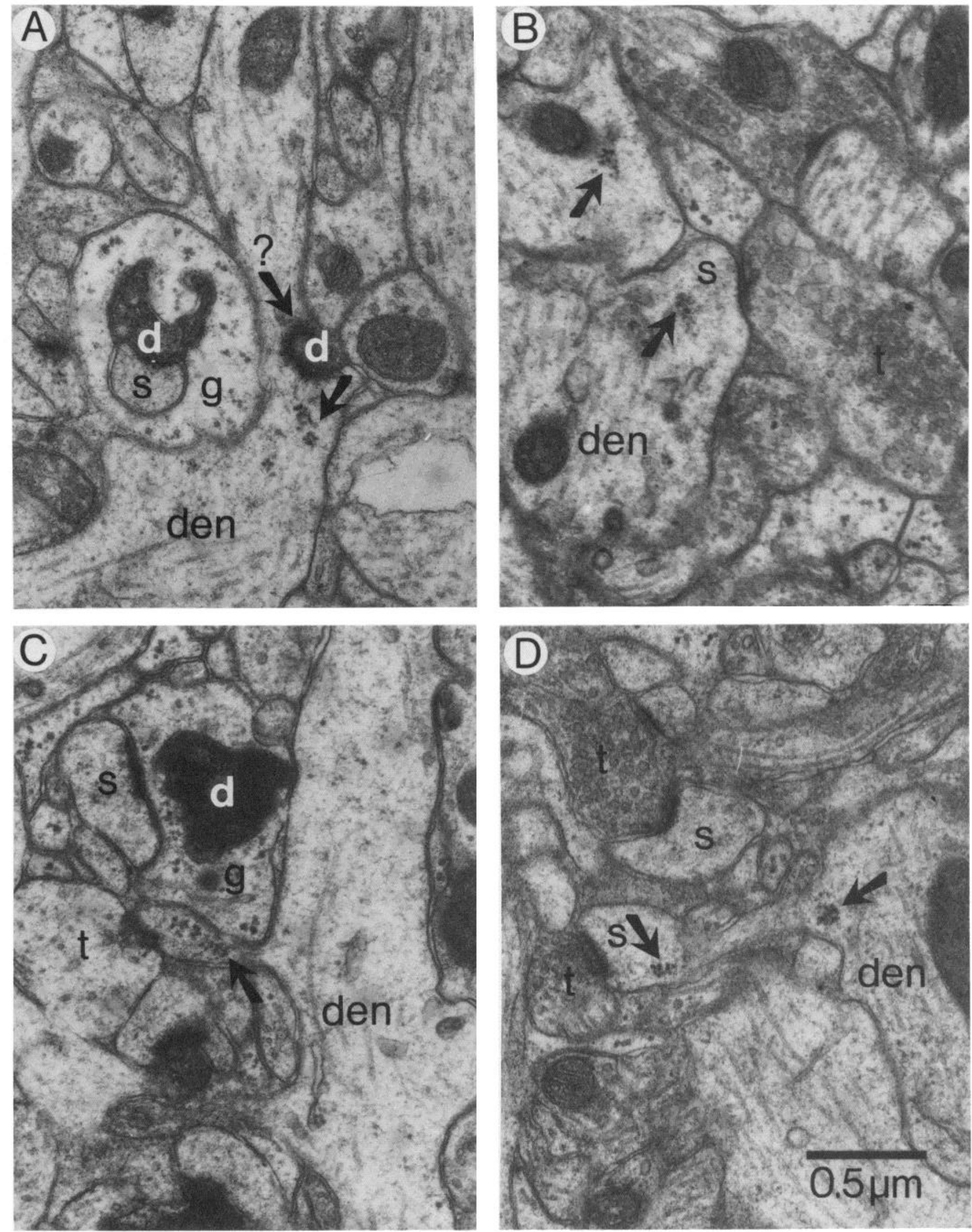

Figure 2. Polyribosomes under collapsed spines and in spine necks and heads. $A$ illustrates polyribosomes adjacent to what appears to be a postsynaptic specialization curving into the main dendrite. This specialization is apposed to a degenerating presynaptic terminal $(d)$. Another degenerating terminal apposed to a spine is entirely surrounded by a glial profile $(g)$. $B$ to $D$ illustrate polyribosomes under and within spines apposed to nondegenerating terminals. Note the large vesicles in the terminal in $B$ and the lack of a large number of vesicles in the terminal in $C$. Note that the spine in $D$ contains polyribosomes not only at the base but also within the spine head. All of these examples come from animals sacrificed 6 days post-lesion. Abbreviations are as in Figure 1. 
During the period of dendritic deterioration and subsequent remodeling, the polyribosomes seem to be localized in essentially the same sites as in normal dendrites, although these sites have a decidedly abnormal appearance. As illustrated in Figure 1, $C$ to $F$, the polyribosomes may be found under profiles which can be identified as spines and under the same sorts of mounds which usually represent the base of spines in normal material. During the early post-lesion interval ( 2 to 6 days post-lesion) the polyribosomes are most often localized under spines contacted by a degenerating presynaptic terminal (Fig. 1, $C$ and $D$ ) or under the irregular mounds apposed to degenerating terminals (Fig. $2 A$ ) which may be collapsed spines. After 8 days post-lesion, polyribosomes are more frequently found under spines with nondegenerating contacts and under stubby spines with thin postsynaptic specializations (Fig. 2, $B$ and $C$ ). Except for the presence of a poorly developed region of membrane specialization, some of these immature-appearing stubby spines are virtually indistinguishable from the mounds which usually represent the base of mature dendritic spines in control material (see, particularly, Fig. $2 B$ ).

While the basic distribution of polyribosomes in denervated dendrites was essentially comparable to that in control material, two differences were apparent even in the qualitative observations. First, the incidence of polyribosomes under spine bases was much higher than in control material, particularly at 6 and 8 days post-lesion. Second, in contrast to the situation in control material, where polyribosomes rarely are observed in spine necks or heads (Steward and Levy, 1982), polyribosomes often were observed in spine necks and heads in the denervated neuropil. Sometimes the polyribosomes were located in spine heads at some distance from the parent dendrite (Fig. 2D), and in some cases, polyribosomes were located both at the base and within the head of a single spine (Fig. 2D). These qualitative impressions provided the background and impetus for the quantitative analyses below.

\section{Quantitative evaluations of polyribosomes in denervated/reinnervated dendrites}

The quantitative analyses were designed to define the extent and time course of any changes in (1) the incidence of polyribosomes under spine bases, (2) the incidence of polyribosomes in spine necks or heads, and (3) the total number of dendritic polyribosomes within the denervated zone.

Polyribosomes under spine bases. The first of these issues was addressed utilizing procedures identical to those which we developed for analyzing the normal incidence of polyribosomes under the base of dendritic spines (Steward and Levy, 1982). At each post-lesion interval, an individual who was not involved in the data analysis (Ms. S. L. Vinsant) was asked to photograph 21 to 22 spine neck/dendritic shaft intersections from the inner half of the denervated portion of the neuropil (approximately the middle molecular layer). The spine neck/dendritic shaft intersection was defined as a dendritic shaft (distinguished by the presence of mitochondria and parallel microtubules) with a protruding thin process with a postsynaptic specialization or containing a spine apparatus. If there was any doubt about whether a given spine neck was connected to a dendrite, the intersection was not included in the sample. While only one intersection was singled out for photography, others meeting the selection criteria usually were present in the field of approximately $60 \mu \mathrm{m}^{2}$, and these were included in the sample. The intersection was considered positive for polyribosomes if more than two ribosomes with a spacing typical of polyribosomal rosettes lay under the base of the spine or within the spine.

In our previous evaluation of the control sides of five animals sacrificed at various times after unilateral entorhinal cortical lesions, an average of $12.2 \pm 1.5 \%$ of the identified spines had polyribosomes (Steward and Levy, 1982). In the present expanded sample, which included these five animals and seven others, an average of 11.6 $\pm 3.9 \%$ had polyribusomes. As illustrated in Figure $3 A$, the incidence of polyribosomes increased dramatically in the denervated dentate gyrus over the post-lesion interval. The incidence of polyribosomes was indistinguishable from control at 2 days post-lesion, slightly elevated at 4 days, and substantially increased between 6 and 10 days, reaching a peak at 8 days post-lesion at a level approximately 3 -fold higher than control. In this and subsequent figures, the average incidence in the control material is indicated by the solid line, and the dashed lines indicate $1 \mathrm{SD}$ around this mean.

The preceding results suggest that there is an increase in the number of spines with underlying polyribosomes during the 6- to 10-day-post-lesion interval. An alternative interpretation, however, is that spines without polyribosomes either disappear or are unrecognizable following denervation to a greater extent than spines with polyribosomes. If spines without polyribosomes were preferentially lost or were unrecognizable, then the increased incidence could reflect simply a decrease in the reference population from which the incidence figure is calculated. To evaluate this possibility, I determined the total cross-sectional area of neuropil which was evaluated in each case, counted the total number of polyribosomecontaining spine bases within that area, and calculated the number of polyribosome-containing spine bases per $1000 \mu \mathrm{m}^{2}$ of neuropil at each post-lesion interval. The results of this evaluation are illustrated in Figure $3 B$. As is evident, there is an increase in the total number of polyribosome-containing spine bases per $1000 \mu \mathrm{m}^{2}$ of neuropil between 6 and 10 days post-lesion which parallels the increase in the incidence of polyribosome-containing profiles.

As indicated above, the incidence calculations can be faulted if spine bases with polyribosomes are preferentially preserved or are more easily recognizable following lesions than spine bases without polyribosomes. On the other hand, the counts of the total number of polyribosome-containing spine bases can be faulted since all spines in the denervated neuropil are somewhat abnormal and difficult to recognize. Therefore, some spine bases might be missed in the counts. Since there is no evidence that spines without polyribosomes are preferentially lost, and since there are parallel increases in the number of polyribosome-containing spine bases per area of neuropil despite a priori expectations that some might 
A

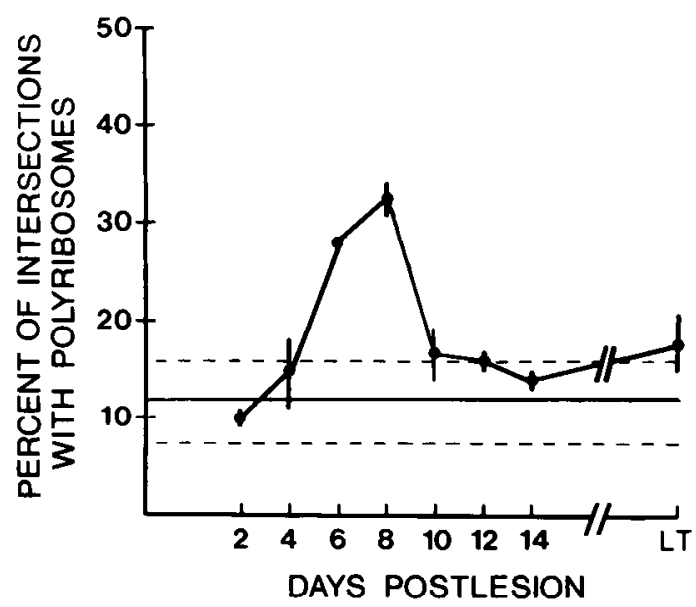

B

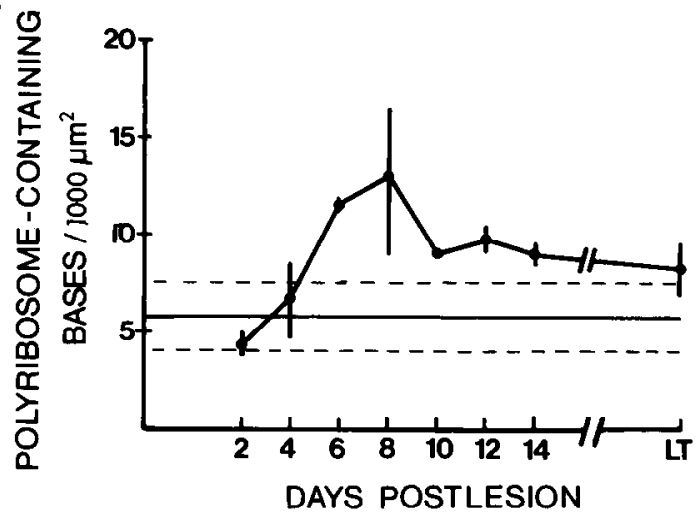

C

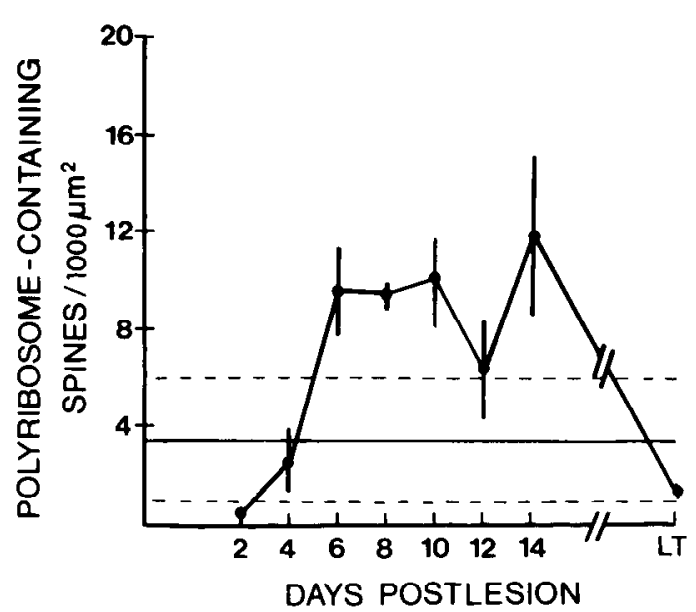

Figure 3. Quantitative analyses of the polyribosomes associated with spines in the denervated neuropil. $A$ illustrates the time course of the increases in the incidence of polyribosomes under the base of dendritic spines (the number of identified spines with underlying polyribosomes per total number of identified spines). $B$ illustrates the time course of the increases in the total number of identified spines with underlying polyribosomes per area of neuropil (see "Materials and Methods" for the criteria for an identified spine). $C$ illustrates the time course of the changes in the total number of polyribosome-containing spine necks and heads per area of neuropil. In each graph, the solid line indicates the mean control value and the dashed lines indicate $1 \mathrm{SD}$ around this mean. The bars indicate the range of values in the two animals examined at each post-lesion interval. be missed, the weight of evidence suggests substantial increase in the number of spine bases with polyribosomes during the 6- to 10-day-post-lesion interval.

In the above analyses, identified spines were considered positive for polyribosomes if the polyribosomes lay under the base of the spine or within the spine. In control material, the vast majority of identified spines are positive by virtue of polyribosomes under the base, and in only a few cases could the polyribosomes also be scored as lying within the spine. In the denervated neuropil, however, this distinction is not straightforward, since many spines are collapsed and are quite stubby. Particularly at 6 and 8 days post-lesion, polyribosomes within a mound (which normally would represent the base of a spine) can also be scored as lying within the spine. The question therefore arose whether the changes reflected an increase in polyribosomes within spines.

Polyribosomes in spine necks or heads. To evaluate whether there were increases in polyribosomes within spines, the same series of 21 to 22 photographs was used as described above. Counts were made of all spine necks or heads which contained polyribosomes, whether or not these spine necks or heads were contiguous with a dendritic shaft (thus, some of these profiles are also the identified spines of the preceding section). The number of polyribosome-containing spine necks and heads per $1000 \mu \mathrm{m}^{2}$ of neuropil then was determined. Spine necks were defined as thin processes extending from spine bases (see above), and spine heads were identified by the presence of a postsynaptic specialization.

As illustrated in Figure $3 C$, there was a clear increase in the number of polyribosome-containing spine necks and heads in the denervated neuropil. As was true of the polyribosome-containing spine bases, the increases were first apparent at 6 days post-lesion. However, in contrast to the increases observed in the polyribosomes under spine bases, the number of polyribosome-containing spine necks and heads remained high at 10 to 14 days post-lesion. Indeed, there appeared to be a secondary peak in the number of polyribosome-containing spine necks and heads at 14 days post-lesion, although the significance of this result is open to question given the rather small numbers and large differences between animals.

The preceding analysis focused on polyribosomes in the spine itself in a position nearer to the postsynaptic specialization than would normally be the case. One interpretation is that polyribosomes which would normally be in the main dendrite move into the spine proper following denervation. It is important, however, to again recall the collapse and deformation of spines in the denervated neuropil. Some of the increases of polyribosomes in spine necks and heads could simply reflect the deformation of the spine rather than a movement of polyribosomes into the spine. In many cases, however, the polyribosomes were clearly at some distance from the spine neck/dendritic shaft intersection and were often within spines which did not appear collapsed (see, for example, Fig. $2 F$ ). Furthermore, some polyribosomal clusters were much closer to the postsynaptic membrane specializations than to the spine neck/dendritic shaft intersection. Thus, the collapse and deformation of spines 
may account for some, but certainly not all of the increases of polyribosomes in spine necks and heads.

Some of the spine necks and heads are also identified spines (see above) and thus there is an overlap between the present analysis and the analysis of the incidence of polyribosomes under spine bases. It is important to recall this potential redundancy in order to avoid over-interpreting the data. Because of the collapse of spines, an increase in polyribosomes within spines would result in an increase in the incidence of polyribosomes under spine bases (since the bases actually represent the entirety of the collapsed spine). Similarly, an increase in the incidence of polyribosomes under spine bases could result in an increase in the number of polyribosome-containing spines. It is impossible to determine which of these effects is the "real" one, and indeed, the question may be irrelevant. Rather than representing different questions, both analyses probably represent alternative approaches to the same question of the relationship between polyribosomes and spines.

As we noted in our previous study of polyribosomes in normal material, analyses from single sections provide only relative indications of the frequency of polyribosomes in spines, since a single seclion does not reveal all of the area subjacent to the spine or within the spine neck or head. Furthermore, one must be concerned with the possibility that spines become deformed in the denervated neuropil, which might affect analyses of polyribosome incidence. Because of these problems, a limited serial section analysis was undertaken of one of the cases sacrificed at 8 days post-lesion, to provide supplementary evidence that the increases in the incidence of polyribosomes under spine bases and within spines were not simply a function of distortion of the spines within the denervated zone. For this purpose, 20 serial sections were collected, and an area of approximately $210 \mu \mathrm{m}^{2}$ was photographed on each section from a mid-proximodistal location along the dendritic tree. This $210-\mu \mathrm{m}^{2}$ area was reconstructed as a montage of four photographs taken at $\times 10,000$, covering an area of approximately $7 \times 30 \mu \mathrm{m}$. From this serial montage, a total of 35 spines were identified where the neck and base were through-sectioned. Three of these spines were not sectioned through the head region but were identified as spines based on the presence of a spine apparatus. Of this population of 35 spines, $15(40 \%)$ had polyribosomes underlying the base, and $7(20 \%)$ had polyribosomes in the spine base or head region. Thus, a total of $60 \%$ of the spines had polyribosomes associated with them. In our previous analysis of normal material which was performed in exactly the same fashion, 34 through-sectioned spines were identified. Only 10 of these $(29 \%)$ had polyribosomes underlying the spine base. Only one spine had polyribosomes in the neck or head, and this spine also had a polyribosome cluster under the spine base. This serial section analysis suffers from the problem of small sample size. Thus, the estimates of the actual incidence of polyribosomes may not be accurate in detail. Nevertheless, the interpretations drawn from the quantitative analyses of single sections are fully substantiated, making it unlikely that the changes in polyribosome frequency result from some change in the configuration of spines in the neuropil arising from deformation.
Dendritic polyribosomes. The preceding results reveal that there is an increase in the number of polyribosomes under spine bases and within the spine proper in the denervated neuropil. Polyribosomes are found in both locations in control material, however, and thus it was of interest to ask if the increases reflected an overall increase in dendritic polyribosomes or a change in their relative distribution. Two analyses were undertaken to address this question. In the first, the total number of dendritic polyribosomes was determined per cross-sectional area of neuropil $\left(1000 \mu \mathrm{m}^{2}\right)$. This measure provides an indication of the overall density of dendritic polyribosomes in a neuropil comprised of dendrites, degenerating and intact terminals, and glia. This measure does not, however, provide any information about the "concentration" of polyribosomes in dendrites, since the proportion of the neuropil which is comprised of dendritic processes changes over the post-lesion interval (Caceres and Steward, 1982). The second analysis was designed to evaluate the number of polyribosomes per cross-sectional area of dendrite. For this analysis, the total volume density of dendritic profiles was measured within the same series of photographs described above, dendritic polyribosomes were counted, and the average number of polyribosomes per $1000 \mu \mathrm{m}^{2}$ of dendrite was determined at each post-lesion interval. In both analyses, polyribosomes in spine bases and in spine necks and heads were included in the counts, but the spine itself was not included in the measures of dendritic cross-sectional area except in the case of the few spines which were contiguous with a dendritic process in the single section. In addition, for the dendritic polyribosomes, the criteria for counting were slightly more stringent, requiring three ribosome-like granules in a cluster with a spacing typical of polyribosomes.

As illustrated in Figure $4 A$, counts of the total number of dendritic polyribosomes per $1000 \mu \mathrm{m}^{2}$ of neuropil indicate a slight increase at 6 and 8 days post-lesion, but these increases are small relative to the variability. Figure $4 B$ illustrates that there is also a slight increase in the number of polyribosomes per $1000 \mu \mathrm{m}^{2}$ of dendrite, but this increase is even less convincing than the increase in number of dendritic polyribosomes per $1000 \mu \mathrm{m}^{2}$ of neuropil. Taken together, these analyses suggest at most only slight increases in the total number of dendritic polyribosomes which certainly do not seem sufficient to account for the dramatic increases in polyribosomes in spin€ bases and within the spine proper.

\section{Discussion}

All of the quantitative analyses suggest that between 6 and 10 days post-lesion, there is an increase in the number of polyribosomes associated with the portion of the postsynaptic cell which is specialized for the receipt of a synaptic contact (namely, the spine and the postsynaptic membrane specialization). The incidence of polyribosomes under identified spine bases increased about 3fold, the number of polyribosome-containing spine bases per unit area increased about 2-fold, and the number of polyribosome-containing spine heads and necks increased about 3-fold. Each of these quantitative analyses can, of course, be faulted on methodological grounds, and it is essential to consider some of the potential problems 
A

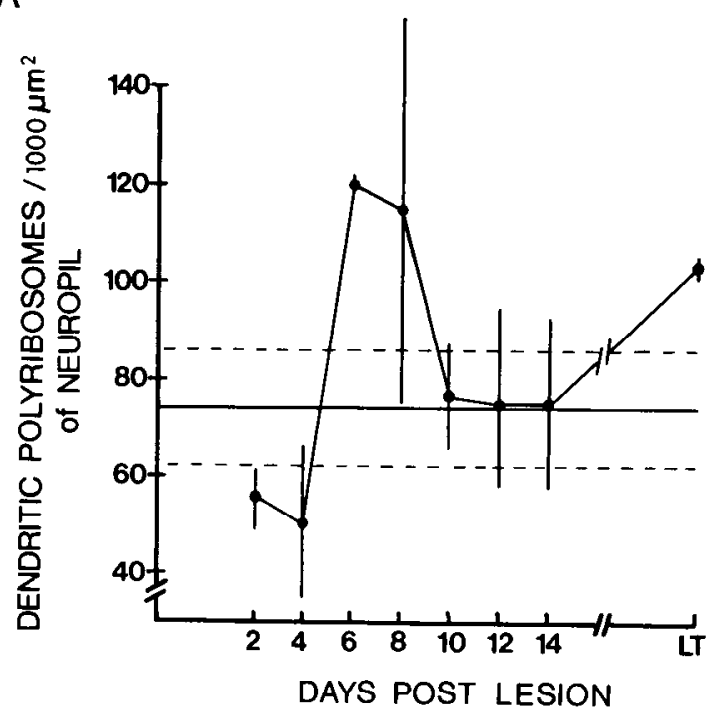

B

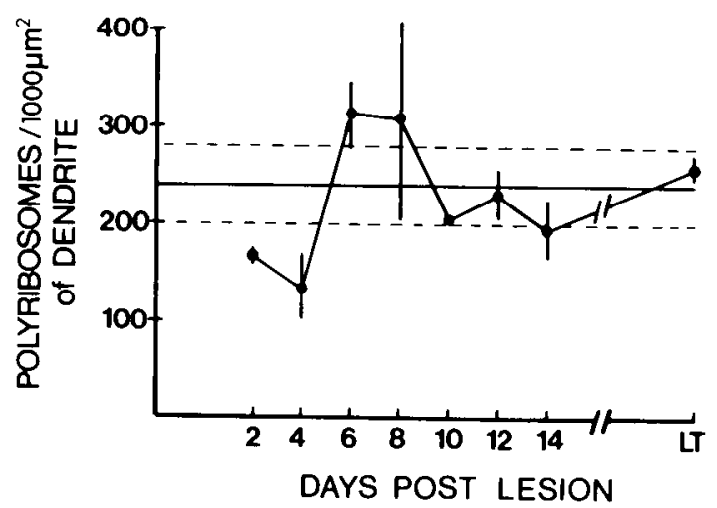

Figure 4. Quantitative analyses of dendritic polyribosomes in the denervated neuropil. $A$ illustrates counts of the total number of dendritic polyribosomes per area of neuropil across the post-lesion interval. $B$ illustrates counts of the total number of dendritic polyribosomes per area of dendrite. The solid line indicates the mean control value and the dashed lines indicate $1 \mathrm{SD}$ around this mean. The bars indicate the range of values at each post-lesion interval.

associated with these analyses prior to discussing the potential significance of the observations.

The first problem which must be considered with respect to all of the analyses is the problem of shrinkage in the denervated neuropil. As has been documented in numerous previous studies (see, for example, Lynch et al., 1975), destruction of the entorhinal cortex results in a considerable shrinkage of the molecular layer of the dentate gyrus. The total height of the molecular layer is reduced by about $20 \%$ at long post-lesion intervals, and it is not known whether this reduction occurs exclusively within the denervated zone or whether it reflects a general collapse. Some of the quantitative analyses could be affected by such a shrinkage (for example, the measurements of the number of polyribosome-containing spines and the number of polyribosomes per area of neuropil). If the lesions remove most of the presynaptic elements resulting in a collapse of dendrites, then the polyribo- some-containing spines on these dendrites and the polyribosomes would be compressed into a smaller area. This sort of collapse could thus artifactually increase the density of polyribosome-containing spines and dendritic polyribosomes per unit area. There are three reasons to believe that this sort of artifact cannot account for the present observations. First, the time course of shrinkage does not correspond to the time course of the changes in polyribosomes. The increases in polyribosomes were first observed at 6 days post-lesion and were maximal at 8 days. Shrinkage, on the other hand, is minimal at 6 and 8 days and does not reach its fullest extent until about 15 days post-lesion (Lynch et al., 1975). Thus, the increases in polyribosomes occur well before there is any substantial shrinkage, and these increases in polyribosomes disappear during the time that most of the shrinkage occurs ( 8 to 15 days post-lesion). Second, the shrinkage, even at its maximal extent, could not result in the extent of the increases which were actually observed. Assuming that shrinkage occurs exclusively in the denervated zone, the $20 \%$ collapse of the entire molecular layer would represent approximately a $30 \%$ collapse in the denervated zone. Assuming that the elements (polyribosomes) which were normally found in the molecular layer would be compressed into a $30 \%$ smaller area, this would mean an increased density of about $43 \%$. In fact, the observed increases are substantially greater than this. Finally, one of the correlated measures (the proportion of polyribosome-containing spine bases) would not be affected by shrinkage, since it is a ratio of the number of polyribosome-containing spine bases to the total number of bases observed. Shrinkage could increase the total number of bases per unit area, but unless there were a concurrent change in the proportion of bases with polyribosomes, the incidence measure would be unchanged. For these three reasons, it seems unlikely that shrinkage could account for any of the changes in polyribosome distribution which were observed.

There are, however, other problems with each of the quantitative measures, some of which have been mentioned under "Results." For example, the measure of the relative incidence of polyribosomes in spine bases is problematic because of the possibility that the identification of spine bases is different in the denervated neuropil. It seems clear that some of the spines disappear following denervation (Parnavelas et al., 1974), and it is possible that spines with polyribosomes are preferentially preserved. In this case, the incidence of spines with polyribosomes could increase artifactually. The selective preservation of spines with polyribosomes cannot, however, account for the increases in the incidence of polyribosome-containing spines for three reasons. First, as noted under "Results," the increase in the incidence of polyribosomes under spine bases is accompanied by an increase in the number of polyribosome-containing bases per unit area of neuropil. This result would not be expected if the increases in incidence came about because of the loss of spines without polyribosomes. Second, the time course of the increase does not correspond to the time course of the spine loss. Parnavelas et al. (1974) report spine loss by 5 days post-lesion (the earliest postlesion interval which was evaluated). Our own studies of spines following entorhinal cortical lesions indicate that 
spine loss occurs by 2 to 4 days post-lesion (Caceres and Steward, 1982). The changes in the incidence of polyribosomes do not become apparent until 6 days post-lesion. Finally, even if only the spines without polyribosomes disappeared, while spines with polyribosomes were preserved, the resulting change in the incidence of polyribosome-containing spines would not account quantitatively for the increases which were actually observed. In the original study of changes in spines in the dentate gyrus, Parnavelas et al. (1974) reported about a 35\% loss of spines. Our counts suggest a somewhat greater loss, so that at 4 days post-lesion, spine density is about $60 \%$ of control (Caceres and Steward, 1982). Assuming a $40 \%$ loss and a normal incidence of about $12 \%$, a complete sparing of spines with polyribosomes would result in an increase in polyribosome incidence to $20 \%$. In fact, the incidence of polyribosomes under spine bases increased to over $30 \%$ at 8 days post-lesion.

A different set of problems arises regarding the interpretation of the changes in the number of polyribosomecontaining spine necks and heads. Here, the major problem is the collapse of the spine into the dendrite, which deforms the spine and brings the contact region much closer to the dendritic shaft. If the polyribosomes are found in the spine proper or near the contact region, it is not possible to determine whether the polyribosomes migrated into the spine or whether the spine collapsed around the polyribosomes. What does seem clear is that the end result would be that the polyribosomes would be situated much closer to the postsynaptic specialization than normal, inviting the speculation that any proteins being synthesized would have easy access to the synaptic region. Furthermore, since the polyribosomes which were scored as being in spine necks and heads were not in the main dendrite, the observations do suggest at least a slight migration of the polyribosomes from the spine neck/dendritic shaft intersection into the spine. While each of the quantitative analyses has certain shortcomings, the shortcomings are different in each situation, yet the indications are consistent, namely, that there is an increase in the number of polyribosomes in or near the region of the dendrite which is specialized for the receipt of an afferent synapse. Similar increases of polyribosomes in or near spines have been briefly noted in previous ultrastructural studies of reinnervation (McWilliams and Lynch, 1978), and in this situation, McWilliams and Lynch reported no detectable shrinkage of the denervated neuropil.

The analyses which sought to determine whether there were also increases in the total number of polyribosomes in the dendrites were more equivocal. The number of dendritic polyribosomes per area of neuropil and the number of polyribosomes per area of dendrite did increase slightly at 6 to 8 days post-lesion, but these increases were quite modest. The dramatic increases in the number of polyribosomes in and around spines, in the face of very modest increases in the total number of dendritic polyribosomes, suggest that the major effect is a redistribution of the polyribosomes into positions closer to the spines.

Significance of the changes in polyribosomes for the reinnervation process. The summary illustrations of Fig-
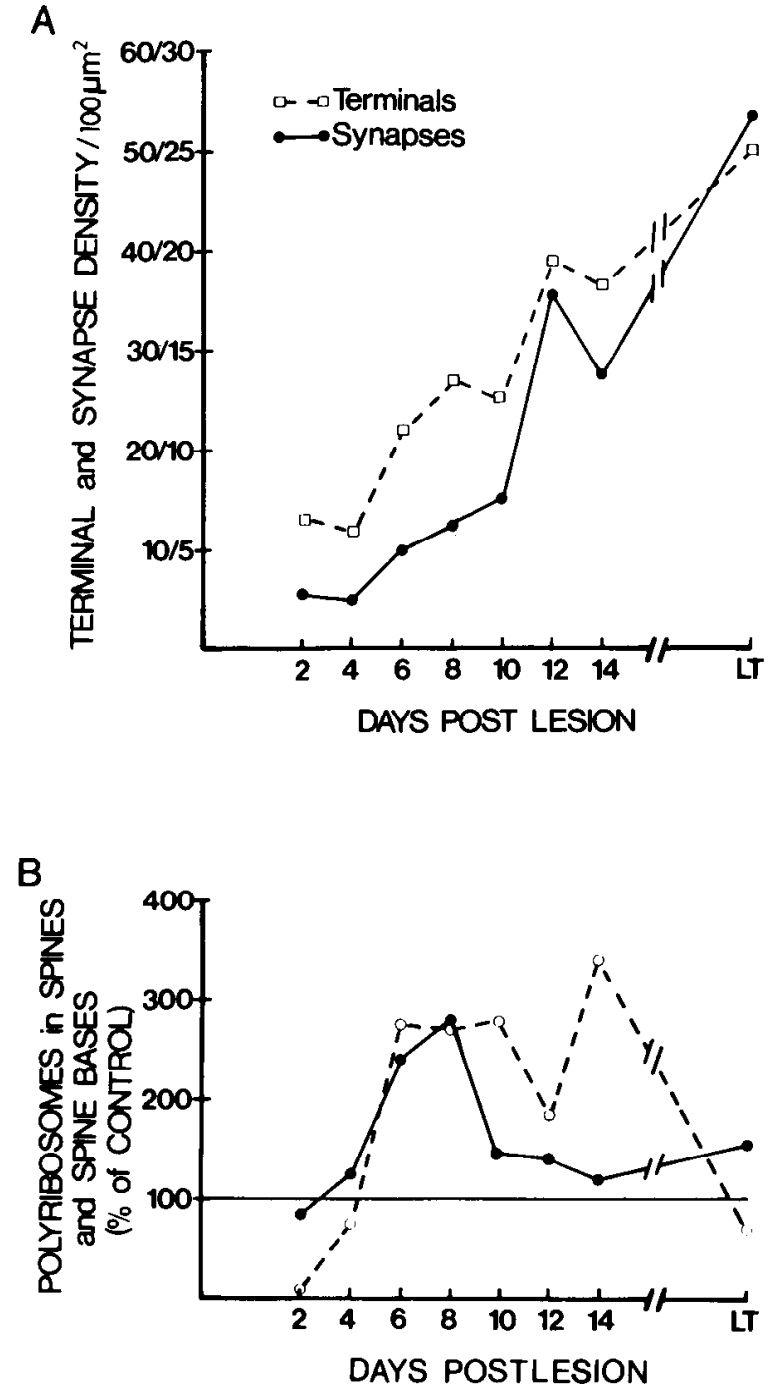

Figure 5. The temporal relationship between terminal proliferation and reactive synaptogenesis and changes in polyribosome-associated spines. $A$ illustrates the time course of the increases in terminal and synapse density in the denervated neuropil of the dentate gyrus after complete destruction of the ipsilateral entorhinal cortex (from Steward and Vinsant, 1982). $B$ illustrates the time course of the increases in polyribosomes under the base of spines (solid lines) and within spine necks and heads (dashed line) in the denervated neuropil.

ure 5 indicate the striking correspondence between the changes in polyribosomes and the terminal proliferation and reactive synaptogenesis in the denervated zone, as revealed by quantitative electron microscopic analyses (from Steward and Vinsant, 1982). It is, of course, conceivable that these parallels are pure coincidence and that the changes in polyribosomes are unrelated to the reinnervation process. We feel, however, that these sorts of relationships are unlikely to arise by chance and more likely signify a relationship between the processes. If this is so, then the question becomes what this relationship might be. At least two possibilities come to mind. First, the reinnervating fibers might be responsible for inducing the changes in the dendrites being reinnervated. Second, the changes in dendrites might occur independently, perhaps reflecting some response of the postsynaptic cell 
to denervation which either induces or permits the reinnervation. A consideration of the time courses of the various processes allows some refinement of the range of possibilities.

We have argued elsewhere (Steward and Vinsant, 1982) that the proliferation of presynaptic processes (terminal proliferation) occurs prior to the time of reinnervation (reactive synaptogenesis). While certain reservations must be kept in mind while evaluating the data, the quantitative analyses suggest that terminal proliferation begins in the middle molecular layer between 4 and 6 days post-lesion, while synapse formation by the reinnervating fibers does not seem to begin until 8 to 10 days post-lesion (see Fig. $5 A$ ). The changes in polyribosomes first became apparent between post-lesion days 4 and 6 , (Fig. $5 B$ ), suggesting that these changes cannot be induced by the reinnervation, since they precede the reinnervation by about 4 days. This suggests that the changes in polyribosomes reflect an independent response of the postsynaptic cell to denervation. What this response signifies is currently a matter for speculation.

Two general possibilities come to mind regarding the possible significance of the changes in polyribosomes. If the polyribosomes are manufacturing some protein(s), these proteins might be involved in some process in the dendrites which prepares them for reinnervation or which induces the reinnervation. In this regard, it is of considerable interest that there appear to be increases in the incorporation of intraveneously administered $\left[{ }^{3} \mathrm{H}\right]$ leucine during the period of altered polyribosome distribution (Fass and Steward, 1981, 1982). Proteins manufactured by the polyribosomes could be involved in the elimination of old areas of membrane specialization, a construction of new postsynaptic membrane specializations (or renovation of old sites), or a rebuilding of any cytoskeletal scaffolding associated with the synapse. Alternatively, proteins manufactured by the polyribosomes might also be involved in inducing the presynaptic growth (terminal proliferation) or the synapse formation by the growing presynaptic processes. One obvious possibility is that the protein(s) may be packaged and released from the spine to diffuse to nearby axons. In this regard, it is of interest that some of the polyribosomal clusters seem to be closely related to cisterns or endoplasmic reticulum under the spine (see, for example, Fig. $1 A$ ). Of course, it may be the construction of new membrane sites or the renovation of old sites which also induces the terminal proliferation and/or reactive synaptogenesis. Obviously, all of these possibilities are speculative at this time, and the crucial next steps involve the identification of the protein(s) being synthesized by the polyribosomes associated with spines and the elucidation of the relationship between the products of polyribosomal synthesis and the process of reinnervation.

Whatever the specific relationship between the reinnervation and the changes in polyribosomes, it is clear that the postsynaptic cell is not simply a passive participant in the reinnervation process. It appears to actively restructure its dendritic specializations (the spines) and certainly alters the cytoplasmic microenvironment around the spine during the period of reinnervation. Whether the reactive changes in the postsynaptic cell are permissive, inductive, or a combination of these, the changes appear to precede the reinnervation and either precede or occur concurrently with the presynaptic growth. It seems reasonable to hope that an understanding of these reactive changes in denervated dendrites will lead to new insights into the cellular and molecular mechanisms of synapse growth in the mature nervous system and perhaps also increase our understanding of how synapses are maintained and modified by functional activity.

\section{References}

Bloom, F. E. (1972) The formation of synaptic junctions in developing rat brain. In Structure und Funclion of Synapses, G. D. Pappas and D. P. Purpura, eds., pp. 101-120, Raven Press, New York.

Caceres, A., and O. Steward (1981) Modifications of granule cell dendrites denervated by entorhinal lesion. Soc. Neurosci. Abstr. 7: 65 .

Caceres, A., and O. Steward (1982) Dendritic reorganization in the denervated dentate gyrus of the rat following entorhinal cortical lesions: A Golgi and electron microscopic analysis. J. Comp. Neurol., in press.

Ebbesson, S. O. E. (1970) The selective silver-impregnation of degenerating axons and their synaptic endings in non-mammalian species. In Contemporary Research Methods in Neu. roanatomy, W. J. H. Nauta and S. O. E. Ebbesson, eds., pp. 132-161, Springer-Verlag, Heidelberg.

Fass, B., and O. Steward (1981) Increases of protein-precursor incorporation in the denervated neuropil of the rat dentate gyrus during sprouting. Anat. Rec. 199: 80A-81A.

Fass, B., and O. Steward (1982) Increases in protein precursor incorporation in the denervated neuropil of the dentate gyrus during reinnervation. Neuroscience, in press.

Globus, A. (1975) Brain morphology as a function of presynaptic morphology and activity. In The Developmental Neuropsychology of Sensory Deprivation, A. Riesen, ed., pp. 9-91, Academic Press, Inc., New York.

Goldowitz, D., W. F. White, O. Steward, G. Lynch, and C. W. Cotman (1975) Anatomical evidence for a projection from the entorhinal cortex to the contralateral dentate gyrus of the rat. Exp. Neurol. 47: 433-441.

Jones, W. H., and D. B. Thomas (1962) Changes in the dendritic organization of neurons in the cerebral cortex following deafferentation. J. Anat. 96: 375-381.

Loesche, J., and O. Steward (1977) Behavioral correlates of denervation and reinnervation of the hippocampal formation of the rat: Recovery of alternation performance following unilateral entorhinal cortex lesions. Brain Res. Bull. 2: 3139.

Lynch, G., G. Rose, C. Gall, and C. W. Cotman (1975) The response of the dentate gyrus to partial deafferentation. In Golgi Centennial Symposium: Perspectives in Neurobiology, M. Santini, ed., pp. 305-317, Raven Press, New York.

Lynch, G., C. Gall, and C. Cotman (1977) Temporal parameters of axon "sprouting" in the brain of the adult rat. Exp. Neurol. 54: 179-183.

Matthews, D. A., C. Cotman, and G. Lynch (1976) An electron microscopic study of lesion-induced synaptogenesis in the dentate gyrus of the adult rat. II. Reappearance of morphologically normal synaptic contacts. Brain Res. 115: 23-41.

McWilliams, R., and G. Lynch (1978) Terminal proliferation and synaptogenesis following partial deafferentation. The reinnervation of the inner molecular layer of the dentate gyrus following removal of its commissural afferents. J. Comp. Neurol. 180: 581-616. 
Parnavelas, J. G., G. Lynch, N. Brecha, C. W. Cotman, and A. Globus (1974) Spine loss and regrowth in hippocampus following deafferentation. Nature 248: 71-73.

Pinching, A. J., and T. P. S. Powell (1971) Ultrastructural features of transneuronal cell degeneration in the olfactory system. J. Cell Sci. 8: 253-287.

Raisman, G. (1969) Neuronal plasticity in the septal nuclei of the adult rat. Brain Res. 14: 25-48.

Steward, O. (1976) Topographic organization of the projections from the entorhinal area to the hippocampal formation of the rat. J. Comp. Neurol. 167: 285-314.

Steward, O. (1982) Events within the sprouting neuron and the denervated neuropil during lesion-induced synaptogenesis. In Changing Concepts of the Nervous System, A. R. Morrison and P. L. Strick, eds., pp. 33-48, Academic Press, Inc., New York.

Steward, O., and B. Fass (1981) Increases in incorporation of protein precursors during reinnervation of rat dentate gyrus are paralleled by increases in the incidence of polyribosomes associated with dendritic spines. Soc. Neurosci. Abstr. 7: 474.
Steward, O., and B. Fass (1982) Polyribosomes associated with dendritic spines in the denervated dentate gyrus: Evidence for local regulation of protein synthesis during reinnervation. Prog. Brain Res., in press.

Steward, O., and W. B. Levy (1982) Preferential localization of polyribosomes under the base of dendritic spines in granule cells of the dentate gyrus. J. Neurosci. 2: 284-291.

Steward, O., and J. Loesche (1977) Quantitative autoradiographic analysis of the time course of proliferation of contralateral entorhinal efferents in the dentate gyrus denervated by ipsilateral entorhinal lesions. Brain Res. 125: 11-21.

Steward, O., and S. L. Vinsant (1982) The process of reinnervation in the dentate gyrus of the adult rat: A quantitative electron microscopic analysis of terminal proliferation and reactive synaptogenesis. J. Comp. Neurol. In press.

Steward, O., C. Cotman, and G. Lynch (1976) A quantitative autoradiographic and electrophysiological study of the reinnervation of the dentate gyrus by the contralateral entorhinal cortex following ipsilateral entorhinal lesions. Brain Res. 114: 181-200. 